ERRATA

\title{
Erratum to: An Alternative Procedure for Selective Leaching of Uranium from Carbonate-Rich Black Shale, Um-Bogma Formation, El-Allouga, Southwestern
}

\author{
A. R. Bakrya,* \\ a Nuclear Materials Authority, El Maadi, Cairo, 11742 Egypt \\ *e-mail: bakrynma@yahoo.com
}

Received March 5, 2021; revised March 5, 2021; accepted March 5, 2021

DOI: $10.1134 / \mathrm{S} 1066362221020168$

Table 6 in page 91 should be replaced by the following:

Table 6. Effect of $\mathrm{pH}$ on the efficiency of selective leaching of uranium from BS

\begin{tabular}{l|c|c|c|l}
\hline \multirow{2}{*}{$\mathrm{pH}$} & \multicolumn{4}{|c}{ Leaching efficiency, \% } \\
\cline { 2 - 5 } & $\mathrm{U}$ & $\mathrm{V}$ & $\mathrm{Cu}$ & $\mathrm{CaO}$ \\
\hline 0.5 & 15.70 & 15.70 & 4.8 & 5.4 \\
0.8 & 14.60 & 14.60 & 4.3 & 4.8 \\
1 & 14.20 & 14.20 & 4.1 & 3.7 \\
1.3 & 13.40 & 13.40 & 3.4 & 3.1 \\
1.8 & 12.30 & 12.30 & 3.2 & 2.7 \\
2.2 & 12.90 & 12.90 & 2.7 & 2.3 \\
2.5 & 12.30 & 12.30 & 2.5 & 2 \\
2.9 & 11.90 & 11.90 & 2.3 & 1.7 \\
3.1 & 14.60 & 14.60 & 1.9 & 1.3 \\
3.6 & 15.50 & 15.50 & 1.7 & 1.00 \\
4 & 16.40 & 16.40 & 1.2 & 0.60 \\
4.5 & 16.80 & 16.80 & 0.5 & 0.50 \\
5 & 17.60 & 17.60 & 0.2 & 0.10 \\
5.7 & 18.90 & 18.90 & 0.00 & 0.00 \\
6 & 15.00 & 15.00 & 1.80 & 0.20 \\
7 & 13.10 & 13.10 & 3.90 & 0.20 \\
8.3 & 11.30 & 11.30 & 5.90 & 0.20 \\
\hline
\end{tabular}

The following references of Reference section should be replaced by:

2. Riegel, M., Tokmachev, M., and Hoell, W.H., J. React. Funct. Polym., 2008, vol. 68, pp. 1072-1080.

https://doi.org/10.1016/j.reactfunctpolym.2008.02.009

3. Singh, D.K., Hareendran, K.N., Sreenivas, T., Kain, V, and Dey, G.K., J. Hydrol., 2017, vol. 171, pp. 228-235.

https://doi.org/10.1016/j.hydromet.2017.05.021

6. Hamza, M.F., El-Aassy, I.E., and Guibalb, E., J. Min. Eng., 2019, vol. 133, pp. 138-148.

https://doi.org/10.1016/j.mineng.2019.01.008

7. Yantasee, W., Fryxell, G.E., Pattamakomsan, K., Sangvanich, T., Wiacek, R.J., Busche, B., Addleman, R.S., Timchalk, C., Ngamcherdtrakul, W., and Siriwon, N., J. Hazard. Mater., 2019, vol. 366, pp. 677-683. https://doi.org/10.1016/j.jhazmat.2018.12.043

12. He, Y., Wang, X., Nie, X., Zou, H. and Pan, N., J. Radioanal. and Nucl. Chem., 2018, 317, pp.1005-1012. https://doi.org/10.1007/s10967-018-5956-4

14. Lu, B.Q., J. Hazard. Mater., 2018, vol. 343, pp. 255-265. https://doi.org/10.1016/j.jhazmat.2017.09.037

33. Kantar, C., and Honeyman, B.D., J. Environ. Eng., 2006, vol. 132, no. 2, pp. 247-255.

https://doi.org/10.1061/(A S CE)07339372(2006)132:2(247)

Table 11 in page 91 should be replaced by the following:

Table 11. Separation of citric acid and calcium citrate using isopropyl alcohol (IPA)

\begin{tabular}{c|c|c|c|c|c|c|c}
\hline \multicolumn{2}{c|}{ Original liquor } & \multicolumn{2}{c}{ Concentrated liquor $(2 \times)$} & \multicolumn{2}{c}{ Highly concentrated liquor $(6 \times)$} & \multicolumn{2}{c}{ Very highly concentrated liquor $(8 \times)$} \\
\hline $\begin{array}{c}\text { water/alcohol } \\
\text { ratio }\end{array}$ & $\begin{array}{c}\text { salted-out Ca } \\
\text { citrate, \% }\end{array}$ & $\begin{array}{c}\text { water/alcohol } \\
\text { ratio }\end{array}$ & $\begin{array}{c}\text { salted-out Ca } \\
\text { citrate, \% }\end{array}$ & $\begin{array}{c}\text { water/alcohol } \\
\text { ratio }\end{array}$ & $\begin{array}{c}\text { salted- out Ca } \\
\text { citrate, \% }\end{array}$ & $\begin{array}{c}\text { water/alcohol } \\
\text { ratio }\end{array}$ & $\begin{array}{c}\text { salted- out Ca } \\
\text { citrate, \% }\end{array}$ \\
\hline $1 / 1$ & 12.00 & $1 / 1$ & 29.00 & $1 / 1$ & 55.00 & $1 / 1$ & 79.6 \\
$1 / 2$ & 29.00 & $1 / 2$ & 45.70 & $1 / 2$ & 72.3 & $1 / 2$ & 93.2 \\
$1 / 3$ & 33.30 & $1 / 3$ & 79.00 & $1 / 3$ & 91.8 & $1 / 3$ & 98.5 \\
$1 / 4$ & 50.10 & $1 / 4$ & 93.40 & $1 / 4$ & 98.4 & - & - \\
$1 / 5$ & 67.10 & $1 / 5$ & 98.00 & - & - & - & - \\
$1 / 6$ & 92.10 & - & - & - & - & - & - \\
$1 / 7$ & 97.90 & - & - & - & - & - & - \\
\hline
\end{tabular}


Figure 6 in page 94 should be replaced by the following:

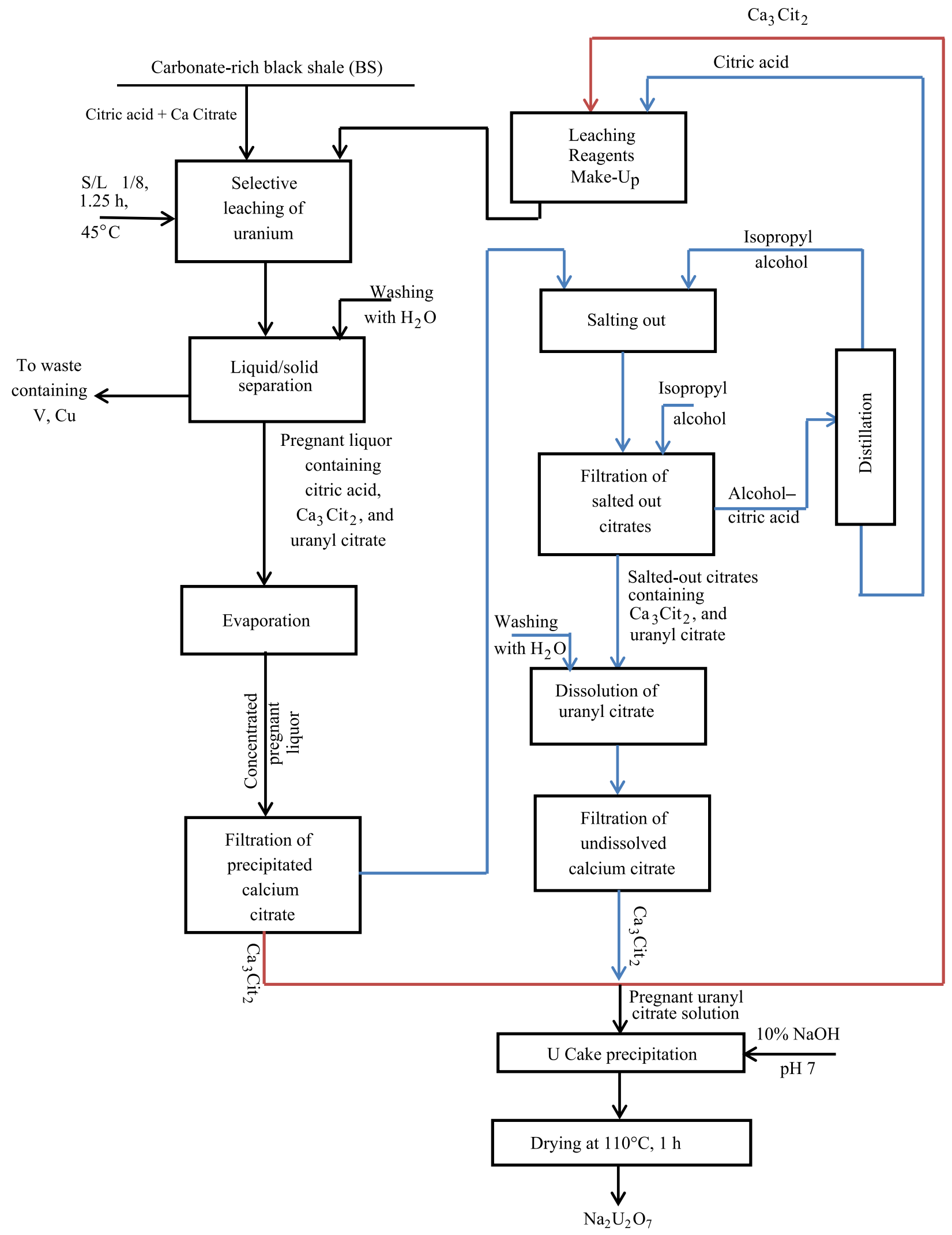

The original article can be found online at https://doi.org/10.1134/S1066362221010136 